\title{
Which Abortion Patients Have Had a Prior Abortion? Findings from the 2014 U.S. Abortion Patient Survey
}

\author{
Rachel Jones, PhD, Jenna Jerman, MPH, and Meghan Ingerick, BA
}

\begin{abstract}
Background: Updated information about abortion patients who have had a prior abortion could inform patientcentered practices that help women avoid unintended pregnancies in the future.

Materials and Methods: Data come from a national sample of 8,380 nonhospital U.S. abortion patients accessing services at 87 facilities. The dependent variable was a self-reported measure of prior abortion. Bivariate and multivariable analyses were used to assess associations between a range of demographic and circumstantial characteristics and reports of obtaining a prior abortion.

Results: We found that $45 \%$ of patients reported having one or more prior abortions. Age was most strongly associated with this outcome, and patients aged 30 and older had more than two times the odds of having had a prior abortion compared with those aged 20-24. Other characteristics associated with an increased likelihood of prior abortion included having one or more children, being black, relying on insurance or financial assistance to pay for the procedure, and exposure to disruptive events in the last 12 months. Characteristics associated with a decreased likelihood of having a prior abortion included having a college degree and living 25 or more miles from the facility where the current abortion was obtained.

Conclusions: Age is the biggest risk factor for having had a prior abortion; the longer a woman has been alive, the longer she is at risk of unintended pregnancy. Some characteristics associated with prior abortion were beyond the control of the individuals experiencing them.
\end{abstract}

Keywords: abortion, repeat abortion, unintended pregnancy

\section{Introduction}

A BORTION IS A common medical procedure undergone by many U.S. women; in 2014 there were 926,000 terminations. ${ }^{1}$ Additionally, many women have more than one abortion over the course of their reproductive lives. In 2008, $50 \%$ of all abortion patients reported having one or more prior abortions, ${ }^{2}$ a proportion that has remained stable since $1993 .{ }^{3}$

There is limited research on the characteristics of individuals who have terminated two or more pregnancies in the United States. Studies based on abortion patients suggest that age and prior births are strongly associated with this outcome, ${ }^{3-6}$ with women aged 30 and older and those who have already had children more likely than younger women and those without children to have had a prior abortion. Compared with patients who are white and college educated, black patients and those who lack a college degree are more likely to be obtaining a second or higher-order abortion. ${ }^{3,5,6}$
The majority of abortion patients was using contraception at the time of conception, including those obtaining secondand higher-order abortions. ${ }^{3,4,6}$ While some studies have found that the latter group was more likely to have been using contraception than women obtaining first abortions, ${ }^{4,6}$ one study also found that women obtaining third- and higherorder abortions were less likely than first-time patients to have been using contraception when they got pregnant. ${ }^{3}$

Prior studies of previous induced abortion are dated ${ }^{3,6}$ or were based on samples restricted to one facility ${ }^{4}$ or one city. ${ }^{5}$ This study uses data from a recent national sample of U.S. abortion patients to determine which characteristics are associated with prior abortion. We build on prior research by including several characteristics in the analyses that have not previously been examined, including distance traveled, exposure to disruptive events, and payment for abortion services. These findings can be used to inform patient-centered clinical practices that help individuals achieve their fertility goals.

Guttmacher Institute, New York, New York.

(C) Rachel Jones et al. 2017; Published by Mary Ann Liebert, Inc. This is an Open Access article distributed under the terms of the Creative Commons Attribution License, which permits unrestricted use, distribution, and reproduction in any medium, provided the original work is properly cited. 


\section{Materials and Methods}

Secondary data for these analyses come from the Guttmacher Institute's 2014 Abortion Patient Survey (APS). The 2014 APS collected information from 8,380 abortion patients accessing services at 87 nonhospital facilities across the United States, and obtained a $76 \%$ response rate. Information was gathered using a four-page, paper-and-pencil, selfadministered questionnaire, available in English or Spanish. Participating facilities were randomly selected, and data are nationally representative of nonhospital abortion patients in the United States. ${ }^{7,8}$ The survey and data collection procedures were approved by the Guttmacher Institute's Institutional Review Board. Detailed information on the data collection and weighting procedures is available in previously published studies. 7,8

The dependent variable for this analysis is a self-reported measure of prior abortion. Two items were used to assess this outcome: half of the sample $(n=4,181)$ was asked the openended question "How many abortions have you had before this one," and half $(n=4,199)$ were asked the closed-ended question "Have you had any abortions prior to this one?" We adopted this strategy to see if the items elicited similar levels of nonresponse and proportions reporting a prior abortion. Some 401 respondents, $4.8 \%$ of the sample, did not answer either item and a significantly larger proportion of women declined to answer the open-ended than closed-ended item $(6.1 \%$ vs. $3.4 \%, p<0.001)$ (not shown). However, similar proportions indicated they had a prior abortion on both measures $(45.2 \%$ vs. $43.4 \%, p=0.05)$. Thus, we dichotomized the more detailed question to make the two measures comparable, and combined them to make one variable.

As a sensitivity analysis, we compared the nonresponders of the continuous item and the dichotomous item for prior abortion; we found no differences between the two groups according to age, race and ethnicity, education, or prior births (not shown). Thus, it is possible that fewer individuals answered the open-ended item simply because it took more effort as opposed to avoiding any stigma that might arise when indicating a specific number of prior abortions.

We examined associations between prior abortion and the following demographic characteristics: age, number of prior births, race/ethnicity, and education, as most of these had been found to be associated with having more than one abortion in prior research. ${ }^{3-5}$ For similar reasons, we included a measure of contraceptive use. Additionally, we assessed for associations between prior abortion and several situational characteristics that could impact women's ability or need to access abortion services one or more times, including method of payment for the abortion, distance from the provider, and exposure to disruptive life events.

The survey included a question about the contraceptive method last used by the respondent and date of use. We distinguished between women who were using a method in the month they got pregnant, those who were not, and those who had never used a method. We adopted this strategy because we were primarily interested in determining whether nonuse or never-use of contraception was associated with having a prior abortion. In preliminary analyses we explored a more detailed measure of type of contraceptive method used but determined that the three-category item allowed for a more straightforward set of associations.
On average, patients travel 15 miles (one way) to access abortion services, ${ }^{9,10}$ and women who live far from a provider may have a harder time accessing services even one time. Thus, we included a measure of distance to facility. Respondents were asked to provide their zip code, and we used this information to estimate how far patients lived from the abortion facility at which they obtained the procedure based on Euclidean, or "straight line," distance. Eight percent of respondents $(n=462)$ did not provide a valid answer to this question, and these respondents were coded to a separate "missing" category so that they would not be excluded from the analyses.

Although most women pay out of pocket for abortion care, ${ }^{7,11}$ those who are able to use health insurance may be able to access abortion services more easily and, in turn, more often. The survey assessed whether women were paying for the abortion themselves, using private or public insurance, or relying on financial assistance to pay some or all of the cost of the procedure; four percent of respondents $(n=278)$ did not answer this item, and rather than exclude them from analysis, we categorized them as a separate group. The survey instrument included a write-in response of "other"; however, closer examination of the responses provided $(n=149)$ suggest that most of these respondents paid out of pocket. Thus, we include these respondents in the self-payment category.

We included a measure of disruptive events as these have the potential to expose women to the risk of repeat unintended pregnancy. Respondents were asked how many of the following they had experienced in the past year: death of a close friend, falling behind on rent/mortgage, separating from a husband/partner, extended unemployment, a dependent or close family member with a serious medical problem, giving birth, the arrest or incarceration of a partner, or having moved more than once. We distinguish between women who had experienced none, one, or two or more of these events.

We first compared the demographic profiles of women who did not answer the item about prior abortions to those who did, using simple logistic regression to assess whether differences were statistically significant. For analyses using the analytic sample, we used simple logistic regression to assess bivariate associations between demographic and situational characteristics and having had a prior abortion. Associations were deemed significant at $p<0.05$. All independent variables were entered into a multivariable logistic regression model.

Because the two items used to measure the dependent variable had slightly different levels of nonresponse, as a sensitivity analysis we generated two separate regression models using the two versions of the dependent variable; these findings are only reported when they differ from the ones using the full sample.

The survey included weights to account for patient nonresponse and variation from the original facility sampling plan, and all analyses were conducted with weighted data, using the "svy" complex sample command prefix within Stata 14.0.

\section{Results}

Five percent of the 8,380 respondents $(n=401)$ did not provide a response to the items about prior abortion (Table 1). Compared with respondents who provided information about 
Table 1. Characteristics of Abortion Patients by Whether or Not They Answered the Item About Previous Abortions and Characteristics of the Full Sample

\begin{tabular}{|c|c|c|c|c|}
\hline \multirow[b]{2}{*}{ Characteristic } & \multicolumn{2}{|c|}{ Missing information } & \multirow[b]{2}{*}{$\mathrm{p}$} & \multirow[b]{2}{*}{ Full sample } \\
\hline & Yes & No & & \\
\hline All & 401 & 7,979 & & 8,380 \\
\hline \multicolumn{5}{|l|}{ Age group } \\
\hline$<20$ & $41(10.3)$ & $945(12.0)$ & NS & $986(11.9)$ \\
\hline $20-24$ & $110(26.8)$ & $2,771(33.9)$ & 0.004 & $2,881(33.6)$ \\
\hline $25-29$ & $101(25.1)$ & $2,134(26.6)$ & NS & $2,235(26.5)$ \\
\hline $30-34$ & $72(18.1)$ & $1,222(15.8)$ & NS & $1,294(15.9)$ \\
\hline$\geq 35$ & $77(19.8)$ & 907 (11.8) & $<0.001$ & $984(12.1)$ \\
\hline \multicolumn{5}{|l|}{ Prior births } \\
\hline 0 & $128(32.1)$ & $3,276(41.1)$ & 0.002 & $3,404(40.7)$ \\
\hline 1 & $102(25.4)$ & $2,107(26.3)$ & NS & $2,209(26.2)$ \\
\hline$\geq 2$ & $171(42.6)$ & $2,596(32.6)$ & $<0.001$ & $2,767(33.1)$ \\
\hline \multicolumn{5}{|l|}{ Race } \\
\hline White & $95(23.3)$ & $3,174(39.5)$ & $<0.001$ & $3,269(38.7)$ \\
\hline Black & $116(29.0)$ & $2,214(27.5)$ & NS & $2,330(27.6)$ \\
\hline Hispanic & $152(38.3)$ & $1,888(24.1)$ & $<0.001$ & $2,040(24.8)$ \\
\hline Other & $38(9.4)$ & $703(8.8)$ & NS & $741(8.8)$ \\
\hline \multicolumn{5}{|l|}{ Education } \\
\hline$<12$ th grade & $69(17.4)$ & $955(12.1)$ & 0.006 & $1,024(12.4)$ \\
\hline High school graduate/GED & $124(30.4)$ & $2,339(29.1)$ & NS & $2,463(29.1)$ \\
\hline Some college or associate degree & $136(34.1)$ & $3,167(39.3)$ & 0.048 & $3,303(39.1)$ \\
\hline College graduate or above & $72(18.1)$ & $1,518(19.5)$ & NS & $1,590(19.4)$ \\
\hline
\end{tabular}

Data presented as $n(\%)$.

NS, not significant.

prior abortions, those who did not were more likely to be older than $35(p<0.001)$, have had two or more births $(p=0.002)$, to be Hispanic $(p<0.001)$, and to be less educated $(p=0.006)$. Despite these significant differences, the demographic profile of the analytic sample was comparable to that of the full sample, and we suspect that our findings would not change substantially if information had been provided by these 401 nonrespondents.

Slightly less than half of abortion patients (44.8\%) had a prior abortion (Table 2), and most of the characteristics examined were associated with this outcome. Sixty percent of patients aged 35 and older had a previous abortion, compared with $38 \%$ patients ages $20-24$. Over $50 \%$ of patients who had a prior birth had also had a previous abortion compared with $29 \%$ of nulliparous women.

These variations were maintained in the multivariable analysis. The likelihood of having a prior abortion increased with age and patients in their 30s had two and a half times the odds of having had a prior abortion (odds ratio [OR]: 2.36; confidence interval [95\% CI]: 2.04-2.73 for 30-34; OR: 2.43; 95\% CI: 2.01-2.93 for 35+) compared with those aged 2024 ; odds were substantially lower for those under age 20 (OR: 0.27; 95\% CI: 0.22-0.35). Similarly, women who had one or more births had twice the odds of nulliparous women of having had a prior abortion (OR: $1.73 ; 95 \% \mathrm{CI}$ : $1.53-1.96$ for one birth; OR: 1.86; 95\% CI: 1.64-2.11 for two or more).

Slightly more than half of Black abortion patients had a prior abortion (54\%), higher than any other racial and ethnic group. This association was maintained in the logistic regression model when white patients were the comparison group (OR: 1.76; 95\% CI: 1.51-1.96).
Patients who had graduated from college were less likely to have had a prior abortion than those with high school degree (OR: 0.61; 95\% CI: 0.52-0.72).

Only a small proportion of abortion patients (10\%, not shown) reported that they had never used contraception, and in the multivariable model these women were less likely to report that they had a prior abortion (OR: 0.81 ; 95\% CI: $0.67-$ 0.97). However, this association was tenuous as it was not significant in either of the models generated for sensitivity analyses (not shown).

Several of the circumstances we examined were associated with prior abortion. Compared with patients who paid for the abortion out of pocket, the odds of having had a prior abortion were higher for those who paid for the procedure using (public or private) health insurance (OR: 1.47 ; 95\% CI: $1.29-$ 1.69) and those who received financial assistance (OR: 1.32; 95\% CI: 1.15-1.52).

The incidence of prior abortion decreased as distance to the facility increased. Nearly half of patients who lived less than 25 miles from the facility were obtaining a second- or higherorder procedure $(47 \%-49 \%)$, compared with $32 \%$ of those who lived at least 100 miles away. These associations were maintained in the logistic regression model, and the odds ratios decreased with each increase in distance (OR: 0.75; 95\% CI: $0.64-0.88$ for $25-49$ miles and OR: 0.55 ; $95 \%$ CI: $0.44-0.68$ for $\geq 100$ miles).

The incidence of prior abortion increased with exposure to disruptive events in the last year; $40 \%$ of women who had experienced none of the disruptions had a prior abortion compared with $52 \%$ of women who had experienced two or more. These associations were also maintained in the 
Table 2. Percentage Distribution of Prior Abortion by Selected Characteristics and Adjusted Odds Ratios and 95\% Confidence Intervals From Multivariate Logistic Regression Analyses Assessing the Associations Between Selected Characteristics and Having Had a Prior Abortion, 2014

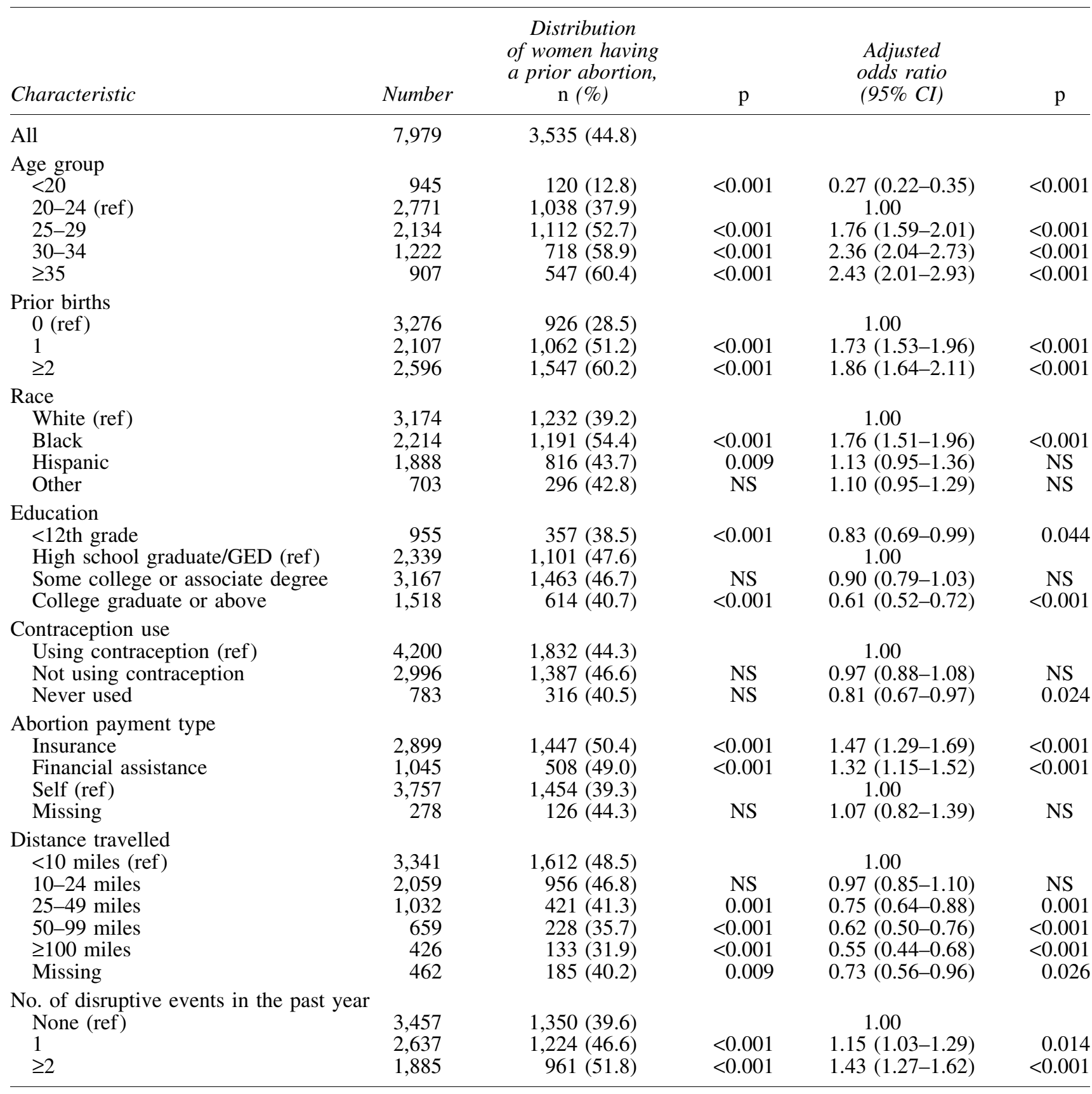

CI, confidence interval; NS, not significant.

multivariable model (OR: 1.15; 95\% CI: 1.03-1.29 for one disruptive event; OR: 1.43 , 95\% CI: $1.27-1.62$ for two or more disruptive events). However, the association between exposure to only one disruptive event was not significant in one of the models in the sensitivity analyses, suggesting that the association, which generated a small odds ratio in the full model, was somewhat tenuous.

\section{Discussion}

Forty-five percent of abortion patients in 2014 had a prior abortion. This was slightly lower than the $50 \%$ found among abortion patients in 2008, and this trend is likely related to the overall decline in abortion incidence. ${ }^{1,12}$

Our findings are in line with prior studies showing that exposure is strongly associated with having a second- or higher-order abortion; ${ }^{3-5}$ incidence of prior abortion was highest for patients aged 35 and older and those with two or more children. The association with age reflects that the older a woman is, the longer her exposure to the risk of pregnancy, including those that are unintended. Women who had multiple prior pregnancies were demonstrably fertile, and therefore at continued risk of pregnancy after a first abortion. 
Associations between race and ethnicity and educational attainment were also consistent with prior studies. ${ }^{3-5} \mathrm{Re}$ lative to white abortion patients, black women were more likely to have had a prior abortion. Black women are also more likely to have unintended births, including multiple unintended births. ${ }^{3}$ These patterns likely reflect larger structural inequalities within healthcare, educational, and economic systems. ${ }^{13}$ That women with college degrees were less likely than high school graduates to have had a prior abortion might represent higher levels of health literacy, which would allow them to better avoid unintended pregnancies.

The incidence of prior abortion was slightly lower for women who had never used contraception than for those who had. This association was significant in the multivariable model that included the full analytic sample, but was not significant in the sensitivity analyses, suggesting it was a weak association. Still, the lack of a positive association suggests that women who have had more than one abortion were not relying on it as their primary method of birth control. In fact, it is likely that many women who had a prior abortion obtained a method of contraception at that time, or at the follow-up visit. ${ }^{3,14}$

Some of the characteristics associated with prior abortion are beyond the control of the individuals experiencing them, and cannot be accounted for in clinicians' counseling and care strategies. Women exposed to disruptive events in the last 12 months were at increased risk of having had a prior abortion. Prior research suggests that some abortions are the result of "rapid repeat pregnancies.",3,15,16 Disruptive events could both lead women to have trouble preventing unintended pregnancies and motivate them to terminate an unintended pregnancy when it occurs. For example, for some women, losing a job may result in lack of access to prescription contraception. Additional disruptions, such as breaking up with a partner, may motivate some women to terminate a pregnancy (intended or otherwise) that they initially planned to carry to term.

Several findings suggest that decreased access to abortion care reduced the "risk" of having more than one abortion. Women who were paying out of pocket for abortion care were less likely than those relying on health insurance or financial assistance to have had a prior abortion. Firsttrimester procedures cost approximately $\$ 500,{ }^{17}$ and threequarters of abortion patients are poor or low income. ${ }^{7}$ Women may only be able to come up with the money to pay for the procedure one time. By contrast, women able to use insurance may not have to scrape money together, making abortion-including second- and higher-order procedureseasier to access. Approximately $14 \%$ of abortion patients rely on financial assistance in the form of subsidies and discounts to pay for their procedures. ${ }^{7}$ When obtaining a prior abortion, women may have learned that financial assistance was available and this may help explain why this payment type was associated with having had a prior abortion.

Distance can be a barrier to abortion. ${ }^{18,19}$ Women who lived 25 or more miles from the facility where they received care were at decreased risk of having had a prior abortion relative to those who lived within 10 miles. One potential explanation is that women who lived further from the facility, where they obtained care, were not necessarily at decreased risk of repeat unintended pregnancy but, rather, were unable to access abortion care multiple times. Alternately, women who lived closer to a facility may have been exposed to knowledge about where to obtain care and, therefore, more likely to have had a prior abortion.

Notably, one implication of the above associations is that the incidence of prior abortion might increase if barriers to abortion were removed; for example, if all women with insurance could use it to pay for abortion care, or if there were more clinics and women did not have to travel so far, more women would be able to access abortion each time they needed it. While "repeat abortion" is sometimes characterized as problematic, ${ }^{20}$ the ability to access abortion care when it is needed-even if more than once-should be prioritized.

Key strengths of this study include its use of a recent, large, national sample of abortion patients. Still, there is the potential for underreporting on this sensitive item; $5 \%$ of the sample did not provide abortion history information, and it is possible these individuals differed from the larger sample in ways in which we are unaware. Given research documenting the underreporting of abortion on surveys of the general population, ${ }^{21}$ it is possible, if not likely, that prior abortions were underreported in this study. This study is also limited by its cross-sectional design, and the range of variables which we were able to assess. For example, we did not examine timing of the index pregnancy in relation to prior pregnancies or abortions, nor did we assess women's reasons for seeking abortions or respondents' relationship with the man involved in the index or prior abortion.

\section{Conclusions}

The findings from this study suggest that continued and expanded access to abortion services is essential for women experiencing an unintended pregnancy. This study also supports previous research suggesting that each abortion is a unique event, occurring in the context of particular situational needs in a woman's life, rather than a repeated behavior. ${ }^{20}$ Several circumstances associated with having a prior abortion, such as age, race, and exposure to disruptive events, are often beyond the control of the individual experiencing them. Additionally, covariates negatively associated with having had a prior abortion reflect possible barriers to accessing care. As such, the results from this study do not necessarily suggest any new or innovative strategies for postabortion contraceptive counseling. For example, while older women are more likely than younger ones to have had a prior abortion, this earlier termination could have occurred decades in the past. Developing a new counseling strategy for abortion patients in their 30s is not likely to reduce the incidence of second- or higher-order abortion among this population. Instead, reducing or eliminating barriers to abortion care may enable those who want or need abortion services, at any point in their lifetime, to more readily access care.

\section{Author Disclosure Statement}

No competing financial interests exist.

\section{References}

1. Jones RK, Jerman J. Abortion incidence and service availability in the United States, 2014. Perspect Sex Reprod Health 2017;49:1-11. 
2. Jones, RK, Finer, LB, Singh, S. Characteristics of U.S. abortion patients, 2008. New York: Guttmacher Institute, 2010.

3. Jones RK, Singh S, Finer LB, Frohwirth LF. Repeat abortion in the United States. New York: Guttmacher Institute, 2006.

4. Prager S, Steinauer J, Foster D, Darney P, Drey E. Risk factors for repeat elective abortion. Am J Obstet Gynecol 2007;197:575.e1-575.e6.

5. Toprani A. Repeat abortions in New York City, 2010. J Urban Health 2015;92:593-603.

6. Westfall JM, Kallail KJ. Repeat abortion and use of primary care health services. Fam Plann Perspect 1995;27: $162-165$.

7. Jerman, J, Jones RK, Onda T. Characteristics of U.S. abortion patients in 2014 and changes since 2008. New York: Guttmacher Institute, 2016;1-28.

8. Jones R, Jerman J. Characteristics and circumstances of U.S. women who obtain very early and second-trimester abortions. PLoS One 2017;1-15. DOI: 10.1371/journal.pone .0169969 .

9. Gerdts C, Fuentes L, Grossman D, et al. Impact of clinic closures on women obtaining abortion services after implementation of a restrictive law in Texas. Am J Public Health 2016;106:857-864.

10. Jones RK, Jerman J. How far Did US women travel for abortion services in 2008? J Womens Health (Larchmt) 2013;22:706-713.

11. Jones RK, Upadhyay U, Weitz TA. At what cost?: Payment for abortion care by U.S. women. Women's Health Issues 2013;23:e173-e178.

12. Jones R, Jerman J. Abortion incidence and service availability in the United States, 2011. Perspect Sex Reprod Health 2014;46:3-14.

13. Gee GC, Walsemann KM, Brondolo E. A life course perspective on how racism may be related to health inequities. Am J Public Health 2012;102:967-974.
14. Kavanaugh M, Jones R, Finer L. How commonly do US abortion clinics offer contraceptive services? Contraception 2010;82:331-336.

15. Baldwin M, Edelman A. The effect of long-acting reversible contraception on rapid repeat pregnancy in adolescents: A review. J Adolesc Health 2013;52:S47-S53.

16. Jacoby M, Gorenflo D, Black E, Wunderlich C, Eyler E. Rapid repeat pregnancy and experiences of interpersonal violence among low-income adolescents. Am J Prev Med 1999;16:318-321.

17. Jerman J, Jones R. Secondary measures of access to abortion services in the United States, 2011 and 2012: Gestational age limits, cost, and harrassment. Women's Health Issues 2014;24:e419-e424.

18. Grossman D, White K, Hopkins K, Potter J. Change in distance to nearest facility and aboriton in Texas, 2012 to 2014. JAMA 2017;317:437-439.

19. Jerman J, Frohwirth L, Kavanaugh M, Blades N. Barriers to abortion care and their consequences for patients traveling for services: Qualitative findings from two states. Perspect Sex Reprod Health 2017. doi: 10.1363/psrh.12024

20. Weitz T, Kimport K. A need to expand our thinking about "repeat" abortions. Contraception 2012;85:408-412.

21. Jones R, Kost K. Underreporting of induced and spontaneous abortion in the United States: An analysis of the 2002 National Survey of Family Growth. Stud Fam Plann 2007; 38:187-197.
Address correspondence to:

Rachel Jones, PhD Guttmacher Institute 125 Maiden Lane New York, NY 10038

E-mail: rjones@guttmacher.org 